\title{
DNA methylation in the hippocampus in rodent and primate models of aging and Alzheimer's disease
}

\author{
Monique Havermans \\ Maastricht University \\ m.havermans@student.maastrichtuniversity.nl
}

\section{Abstract}

With the aging of the world's population, Alzheimer's disease (AD) is a growing problem. It is caused by pathological hallmarks including amyloid $\beta$ plaques and neurofibrillary tangles, but the underlying molecular mechanisms are unknown. DNA methylation, the binding of a methyl group to cytosine in the DNA, has been suggested to play a role in the development of AD. It is catalyzed by the enzyme DNA methyltransferase (DNMT) and creases a 5-methylcytosine $(5 \mathrm{mC})$, which generally represses gene expression. $5 \mathrm{mC}$ can be converted into $5 \mathrm{hmC}$ by ten eleven translocation (TET) enzymes and this is believed to increase gene expression. DNA methylation, DNA hydroxymethylation and DNMT3A, a member of the DNMT family involved in de novo DNA methylation, are suggested to influence cognitive functions, but their exact role in the onset and development of AD remains unknown. In this study, possible changes in these epigenetic markers in the hippocampal sub-regions (cornu ammonis 1,2 and 3 (CA1, 2 and 3 ) and dentate gyrus (DG)) of several animal models for $A D$ are examined. $5 \mathrm{mC}$ levels in the $\mathrm{DG}$ of $\mathrm{J}_{2} \mathrm{O}$ were shown to decrease with aging and Dnmt3a levels in the CA1-2 of these mice showed an age-related increase. In contrast to the J2O mice, $3 \times \mathrm{TgAD}$ mice showed an increase in $5 \mathrm{mC}$ in the DG as well as an increase in Dnmt3a levels. In both the APP/PS1 mouse model and the vervets, no significant increases or decreases were detected. These results show that there are major differences in DNA methylation in hippocampal sub-regions between different animal models for AD.

\section{Keywords}

Alzheimer's disease (AD), DNA methylation, 5-methylcytosine (5mC), 5-hydroxymethylcytosine ( $5 \mathrm{hmC}$ ), DNA methyltransferase za (DNMT3A), hippocampus, cornu ammonis 1 and 2 (CA1-2), cornu ammonis 3(CA3), dentate gyrus (DG), J20 mouse model, APP/PS1 mouse model, 3 XTgAD mouse model, Caribbean vervets. 


\section{Introduction}

As the world's population is aging rapidly, chronic health issues like cognitive decline are a growing problem. This relatively innocent cognitive decline can eventually progress into dementia which is more problematic for everyday life. Alzheimer's disease (AD), a progressive neurodegenerative disorder, is the most common cause of dementia, with symptoms like memory problems, mood swings and confusion, but also personality changes, sleeping problems and depression. In the Netherlands, one in five people will develop AD during their lives and all these people will be in the need of intensive care. Therefore, a method to suppress, cure or even prevent this disease is desirable.

Important pathological features of the brain of an $A D$ patient are aggregation of the amyloid $\beta$ protein and abnormalities in the structural protein Tau, which results in amyloid $\beta$ plaques around the neurons and fibrillary tau tangles inside the neurons. This pathology affects the neurons leading to cellular loss and vascular damage, long before the onset of the first symptoms. The molecular
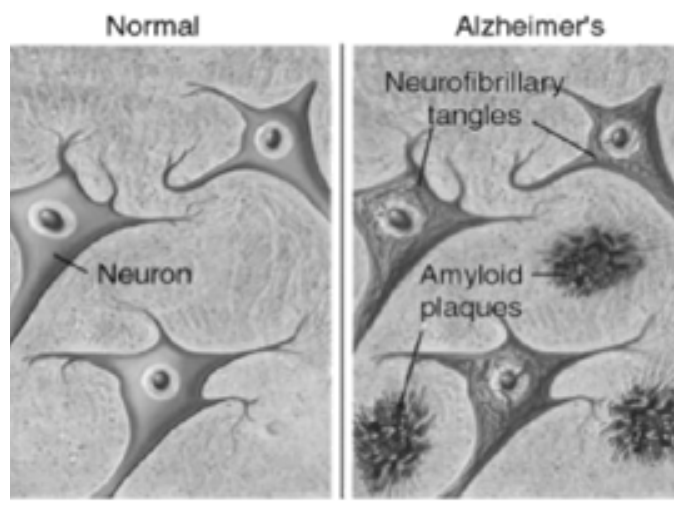

Figure 1. Healthy neurons compared to neurons in the $A D$ brain. mechanisms underlying these processes are not clear and it is not exactly known why one person develops $\mathrm{AD}$ and someone else does not. An explanation has been sought in genetics, but twin studies have pointed out that genes cannot completely explain the origin of the disease. Therefore, a role for the environment has been suggested.

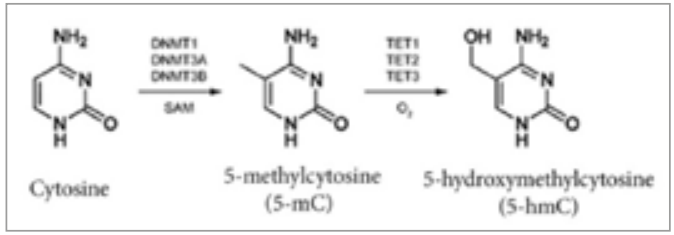

Figure 2. The molecular structures of the cytosine base in the DNA, 5-methylcytosine and 5-hydroxymethylcytosine.
The environment can influence molecular processes in the human body via epigenetic mechanisms. Epigenetics is the study of heritable but reversible changes in gene function that do not originate from a change in the DNA sequence. There are various epigenetic mechanisms which can influence gene expression resulting in altering protein levels in cells. DNA methylation, the most studied and best known 
epigenetic mechanism, is the addition of a methyl group, a small molecule, to the 5 -position of a cytosine nucleotide, creating a 5-methylcytosine $(5 \mathrm{mC})$. This mainly happens at specific parts of the genome, mostly at regulatory regions of the DNA. DNA methylation is catalyzed by an enzyme called DNA methyltransferase (DNMT). There are multiple subtypes of DNMTs, of which one is DNMT3A. DNMT3A catalyzes de novo DNA methylation; the methylation of yet unmethylated sites, while other DNMTs catalyze for example maintenance of existing methylated sites.

As mentioned, DNA methylation has a function in the regulation of gene expression. In general, $5 \mathrm{mC}$ inhibits gene expression by preventing transcription factors from binding and thereby blocking protein expression. However, $5 \mathrm{mC}$ can be converted into 5 -hydroxymethylcytosine $(5 \mathrm{hmC})$ by ten eleven translocation (TET) enzymes. $5 \mathrm{hmC}$ in most cases promotes gene expression by attracting transcription factors.

Not all brain structures are equally affected by the amyloid $\beta$ plaques. One of the most affected brain parts is the hippocampus, an important contributor in learning and memory processes. Any disturbance in this system can lead to problems regarding cognition. Therefore, the hippocampus is one of the brain regions of interest for scientists in the field of $A D$. It consists of four sub-regions; cornu ammonis 1 and 2 (in this report referred to as one, $C A 1-2$ ), cornu ammonis 3 (CA3) and the dentate gyrus (DG). Based on previous studies, these sub-regions seem to show differences in DNA methylation and hydroxymethylation, but no consensus has been reached on this yet.

Today, not much is known about DNA methylation and hydroxymethylation in the brain. Few studies have investigated these epigenetic mechanisms in the brain in AD patients and a lot of these studies targeted different brain regions. One study found decreased levels of both $5 \mathrm{mC}$ and $5 \mathrm{hmC}$ levels in the hippocampus in human $\mathrm{AD}$ patients compared to healthy subjects. Also DNMT3A is a gene of interest in $A D$ research, but previous studies gave conflicting results.

The goal of this study is to examine possible age-related changes in $5 \mathrm{mC}, 5 \mathrm{hmC}$ and Dnmtзa levels in the earlier mentioned sub-regions of the hippocampus of several animal models for $A D$ and to compare the different animal models. This possibly gives more information on the relationship between DNA (hydroxy)methylation and the AD pathology. This is done by the performing a specific staining for $5 \mathrm{mC}, 5 \mathrm{hmC}$ and Dnmt3a by the use of antibodies. 


\section{Material and methods}

\section{Animals and Experimental Design}

Brain sections of three transgenic mouse models were used; J2O, APP/PS1 and 3XTgAD mice. These mice were genetically modified so they had mutations in different genes related to Alzheimer's disease; amyloid precursor protein (APP), presenilin 1 (PS1), microtubuleassociated protein Tau (MAPT or Tau). This leads to an Alzheimer-like pathology in their brains. Next to these mice, brain sections of Caribbean vervets were used. These primates were non-transgenic, meaning that no changes were made to their genome as vervets are known to develop an Alzheimer's-like pathology in their brains naturally. The used animal models and the mutations they contained are depicted in table 1.

Table 1. The used animal models and the mutations they contained.

\begin{tabular}{llll} 
& APP $_{\text {mut }}$ & PS1 $_{\text {mut }}$ & Tau $_{\text {mut }}$ \\
\hline J20 mice & + & - & - \\
\hline APP/PS1 mice & + & + & - \\
\hline 3XTgAD mice & + & + & + \\
\hline Caribbean vervets & - & - & -
\end{tabular}

In J20 mice, amyloid $\beta$ plaques start to develop by five to seven months of age, while the brains of APP/PS1 mice show this plaque formation already at three to four months. Also phosphorylated tau, a precurosor for fibrillary tau, has been seen in APP/PS1 mice, but no fibrillary tau protein like in the natural, human form of AD. 3XTgAD mice show very progressive amyloid $\beta$ aggregation, starting from six months of age, and tau tangles from twelve to fifteen months. The brain sections used in this study originated from mice and vervets sacrificed at different ages. Based on their age, the animals were divided over three age groups as depicted in table 2 . 
Table 2. The experimental design. ( $\mathrm{N}$ : number of mice included in the age group)

\begin{tabular}{lllll} 
Animal model & \multicolumn{1}{l}{ Age group 1 } & Age group 2 & Age group 3 \\
\hline \multirow{2}{*}{ J20 mice } & $\mathrm{N}$ & 8 & 3 & 2 \\
\cline { 2 - 5 } & Age & $4-8$ months & 16 months & 24 months \\
\hline \multirow{2}{*}{ APP/PS1 mice } & $\mathrm{N}$ & 4 & 3 & 2 \\
\cline { 2 - 5 } & Age & 6 months & $16-18$ months & $23-27$ months \\
\hline \multirow{2}{*}{ 3xTgAD mice } & $\mathrm{N}$ & 4 & 3 & 3 \\
\cline { 2 - 5 } & Age & 5 months & $14-17$ months & 27 months \\
\hline \multirow{2}{*}{ Caribbean vervets } & $\mathrm{N}$ & 3 & 5 & 4 \\
\cline { 2 - 5 } & Age & $10.9-14$ years & $14.9-17$ years & 19-32 years
\end{tabular}

\section{Detection of $5 \mathrm{mC}, 5 \mathrm{hmC}$ and Dnmt3a}

Per animal, three series of brain sections, including the hippocampus, were used. These brain sections were stained with a histochemical staining; one series specific for $5 \mathrm{mC}$, one for $5 \mathrm{hmC}$ and one for Dnmt3a. To accomplish this, anti-5mC, anti-5hmC and antiDnmtza antibodies (the primary antibodies) were added to the sections for binding to the molecules of interest. Subsequently, secondary antibodies labeled with the enzyme hydrogen perixode were added for binding to the primary antibodies. Adding a solution with $3,3^{\prime}$-diaminobenzidine (DAB) resulted in the conversion of $D A B$ by hydrogen peroxidase into an insoluble molecular with a dark brown color visible under the microscope. This way, a brown color forms at the places where either $5 \mathrm{mC}, 5 \mathrm{hmC}$ or Dnmt3a is located.

\section{Analysis of $5 \mathrm{mC}, 5 \mathrm{hmC}$ and Dnmt3a immunoreactivity}

For analysis, images of the brain sections were taken with a camera connected to a Bx50 brightfield microscope (Olympus, Tokyo, Japan). Per brain section, nine images were made; one image of the whole hippocampus with a $2 x$ objective (lower magnification) and eight of the different sub-regions of the hippocampus with a $20 x$ objective (higher magnification). 
The images of the sub-regions were analyzed with the software program ImageJ.The amount of $5 \mathrm{mC}, 5 \mathrm{hmC}$ and Dnmt3a present were determined based on the integrated density (IntDen), a variable combining the gray value and the surface. The higher the IntDen, the more $5 \mathrm{mC}, 5 \mathrm{hmC}$ or Dnmt3a was present in the brain section. Processing of the raw data was performed in programming language $R$ (The $R$ Foundation, Vienna, Austria) and RStudio (the Foundation for Open Access Statistics, Boston, MA, USA).
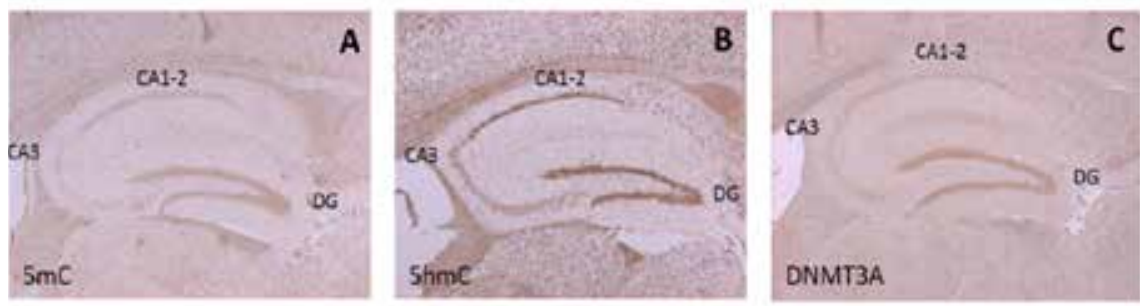

Figure 3. Images of the $5 \mathrm{mC}(\mathrm{A}), 5 \mathrm{hmC}(\mathrm{B})$ and Dnmt3a (C) immunohistochemical stainings showing the whole hippocampus, made with a $2 x$ objective. (5mC: 5-methylcytosine, $5 \mathrm{hmC}: 5$-hydroxymethylcytosine, Dnmt $3 \mathrm{a}$ : DNA methyltransferase 3A, DG: dentate gyrus, CA3: cornu ammonis 3, CA1-2: cornu ammonis 1 and 2)

The IntDen was corrected for the background gray value and all values were normalized per animal model, per sub-region of the hippocampus for the values of the youngest age group. The statistical analysis was performed in Rstudio by the use of a one-way ANOVA with a Bonferroni post-hoc test to compare the individual age groups to each other. The statistical significance was set at $a=0.05$. Graphs were built in GraphPad Prism software (Version 6, GraphPad Software, San Diego, CA, USA).

\section{Results}

To examine the relationship between DNA (hydroxy)methylation in the hippocampus and the development of $A D$ in animal models, hippocampal sections of three transgenic mouse models for $A D$ and non-transgenic aged Caribbean vervets were immunohistochemically stained for $5 \mathrm{mC}, 5 \mathrm{hmC}$ and Dnmt3a. The microscopic images of the whole hippocampus of the three different stainings are shown in figure 3. The results are summarized in table 3. 
Table 3. A summary of the results; the colomns indicate the measured marker ( $5 \mathrm{mC}: 5$-methylcytosine, $5 \mathrm{hmC}$ : 5-hydroxymethylcytosine, Dnmt3a: DNA methyltransferase $3 \mathrm{~A}$ ), the rows indicate the studied mouse model and the hippocampal sub-region (DG: dentate gyrus, CA3: cornu ammonis 3, CA1-2: cornu ammonis 1-2).

$\uparrow$ indicates a significant increase, $\downarrow$ indicates a significant decrease, - indicates no significant increase or decrease and an asterisks indicates a significant increase or decrease between two of the three age groups.

\begin{tabular}{|c|c|c|c|c|}
\hline & & $5 \mathrm{mC}$ & $5 \mathrm{hmC}$ & Dnmt3a \\
\hline \multirow[t]{3}{*}{ J2O } & DG & $\downarrow(p=0.038)$ & - & - \\
\hline & $\mathrm{CA}_{3}$ & - & - & - \\
\hline & CA1-2 & - & - & $\uparrow(p=0.040)$ \\
\hline \multirow[t]{3}{*}{ APP/PS1 } & DG & - & - & - \\
\hline & CA3 & - & - & - \\
\hline & CA1-2 & - & - & - \\
\hline \multirow[t]{3}{*}{$3 \times \operatorname{TgAD}$} & DG & $\uparrow^{*}(p=0.039)$ & - & $\therefore$ \\
\hline & CA3 & - & - & - \\
\hline & CA1-2 & - & - & - \\
\hline \multirow[t]{3}{*}{ Caribbean vervet } & DG & - & - & - \\
\hline & CA3 & - & - & - \\
\hline & CA1-2 & - & - & - \\
\hline
\end{tabular}

In the $\mathrm{J}_{20}$ mouse model, there is an age-related pattern visible for $5 \mathrm{mC}$ levels in the DG. In older mice, the $5 \mathrm{mC}$ levels are significantly lower than in the younger ones $(p=$ 0.038 ) (Figure $4 \mathrm{~A}$ ). The same pattern is seen in the $\mathrm{CA}_{3}$ region, but this is not statistically significant $(p=0.071$ ) (Figure $4 B$ ). There are no significant age-related changes in $5 \mathrm{hmC}$ levels in the J2O mice, but there is a significant increase in Dnmt3a levels in the CA1-2 region $(p=0.040$ ) (Figure $4 \mathrm{C})$.

No clear age-related pattern is seen in either $5 \mathrm{mC}, 5 \mathrm{hmC}$ or Dnmt3a levels in all hippocampal sub-regions in the APP/PS1 mouse models. The same accounts for the Caribbean vervets. $5 \mathrm{mC}, 5 \mathrm{hmC}$ and Dnmt3a levels seem stable in the $\mathrm{DG}, \mathrm{CA} 3$ and $\mathrm{CA} 1-2$ of these primates.

In contrast, $5 \mathrm{mC}$ levels in the DG of the $3 \times \operatorname{TgAD}$ mouse model increase significantly with age $(p=0.039)$. In addition, pairwise comparison indicates a statistically significant different between age group 2 and $3(p=0.049$ ) (Figure $4 D$ ). Also in the $3 \times \operatorname{TgAD}$ mouse 
model, Dnmt3a levels of age group 3 in the DG are significantly higher than those in age group 2 ( $p=0.049$ ) (Figure $4 \mathrm{E})$. The same trend could be present in the $\mathrm{CA}_{3}$ region in these animals, but it is not statistically significant $(p=0.066)$ (Figure $4 \mathrm{~F})$.

${ }^{*}$ Not all results were included in this figure, due to space limitations.

\section{Discussion/Conclusion}

The aim of this study was to examine the relationship between DNA (hydroxy)methylation and the development of $A D$ in the hippocampal sub-regions in several aging animal models for $A D$. In the $D G$ of J2o mice, an age-related decrease in $5 \mathrm{mC}$ levels was detected, while $5 \mathrm{hmC}$ and Dnmt3a levels remained stable in this region. The Dnmt3a levels in the CA1-2 of $J_{20}$ mice increases statistically significant with aging. In contrast to the J2O mice, 3XTgAD mice show a statistically significant increase in both $5 \mathrm{mC}$ and Dnmt3a immunoreactivity in the DG with aging. $5 \mathrm{hmC}$ levels on the contrary, seem to be relatively stable and show no significant increase or decrease in any of the mouse models in any of the hippocampal sub-regions. In the vervets, no significant change in either $5 \mathrm{mC}, 5 \mathrm{hmC}$ or Dnmt3a levels could be detected.
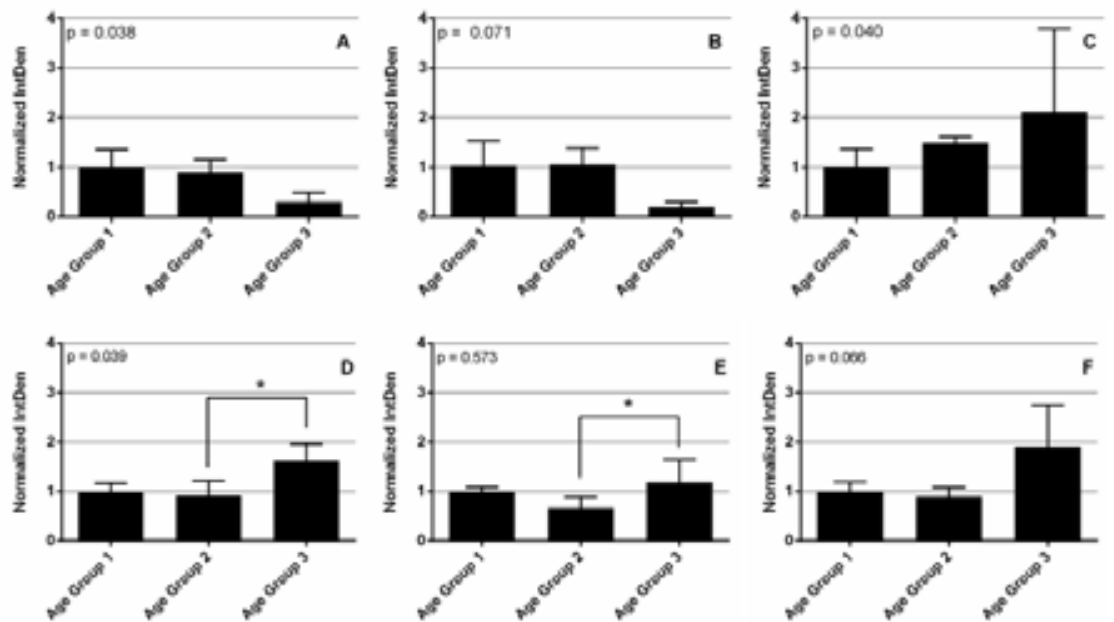

Figure 5. A: The $5 \mathrm{mC}$ levels in the DG of the $\mathrm{J}_{2}$ o mouse model. B: The $5 \mathrm{mC}$ levels in the $\mathrm{CA}_{3}$ of the $\mathrm{J}_{2} \mathrm{O}$ mouse model. C: The Dnmt3a levels in the CA1-2 of the J20 mouse model. D: the $5 \mathrm{mC}$ levels in the DG of the 3xTgAD mouse model. E: The Dnmt3a levels in the DG of the 3xTgAD mouse model. F: The Dnmt3a levels in the CA3 of the 3XTgAD mouse model. (IntDen: integrated density, 5mC: 5- methylcytosine, Dnmt3a: DNA methyltransferase 3A, DG: dentate gyrus, CA3: cornu ammonis 3, CA1-2: cornu ammonis 1 and 2 
The results indicate that there are differences between these four animal models. The most striking difference is the decrease in $5 \mathrm{mC}$ levels in the DG of $\mathrm{J}_{20}$ mice while these levels increase in 3XTgAD mice. The biggest pathological difference between these two models is that the $3 \times \operatorname{TgAD}$ mice develop neurofibrillary tangles, while the J20 mice do not. It thus might be that this pathology has an effect on the DNA methylation patterns in the DG of these mice. However, no previously conducted work could be found on this, making it an interesting subject for further research.

Also, there are some differences between the J20 mouse model and the APP/PS1 mice worth mentioning. The effects of aging on DNA methylation seen in the J2O mouse model seem to be diminished by the additional PS1 transgene present in the APP/PS1 mice. It thus might by that this mutated form of the PS1 gene compensates for the effects on DNA methylation of the mutated APP form in mice. One study reported lower amyloid $\beta$ accumulation in fibroblasts with mutated PS1, but no study has been performed with the PS1 mutation in the used APP/PS1 mouse model.

The vervets, which develop amyloid $\beta$ plaques naturally without the presence of any mutated transgene, do not show age-related changes in either $5 \mathrm{mC}, 5 \mathrm{hmC}$ or Dnmt3a levels. This could imply that the changes in DNA methylation seen in the transgenic mouse models may be a separate downstream effect of their mutated transgenes. It is also possible that these differences are caused by minor differences in AD pathology as the origin of this pathology varies in the used animal models. In addition, even though Caribbean vervets show similar amyloid $\beta$ pathology as humans, its effect on cognition is rather small compared to humans. It could be that this is related to the negligible changes in DNA methylation in the hippocampus compared to mouse models for AD or human $A D$ patients. However, more research is needed on this aspect.

An important question for further research in this field is which animal models are suited for studying DNA (hydroxyl)methylation in the hippocampus in AD and which are not. While the J20 mouse model carries a genome more similar to those common in familial $A D$, the pathology of the $3 x \operatorname{TgAD}$ has more similarities with human AD pathology. One human post mortem study reported lower $5 \mathrm{mC}$ and $5 \mathrm{hmC}$ levels in the hippocampus of $A D$ patients compared to healthy subjects. This pushed the $J_{20}$ mouse model forward as most veracious mouse model for human AD. However, this is based on only one animal study with just four different animal models and one human post mortem study. In addition, conflicting results regarding DNA methylation levels in AD are found in other 
studies who investigated other relevant brain structures. To reach a consensus on this, more research is needed.

While the first amyloid $\beta$ plaques in J20 mice are seen between five to seven months of age, the first staining was performed on mice between four to eight months of age. This means that this study does not include measurements before the onset of the $A D$ pathology and that important data is missing on DNA (hydroxyl)methylation during the onset of the AD pathology. The same accounts for the other two mouse models. In APP/ PS1 mice, plaques are already present at three to four months while the youngest age group includes mice of six months of age. The first amyloid $\beta$ plaques in 3XTgAD mice appear around six months while the youngest age group consists of mice of five months of age. Therefore it could be recommended for further research to include younger mice so that possible epigenetic changes before the onset of the AD hallmarks are included in the measurements.

This study shows that there are major differences in DNA methylation in hippocampal sub-regions between different animal models for $A D$. There seems to be a role for $5 \mathrm{mC}$ in the development of $A D$, as well for DNMT3A based on these animal models. However, not all results are in line with the few previously conducted studies, indicating that DNA methylation is more complex than it may seem. As multiple studies have confirmed that DNA methylation plays a role in AD, future studies can focus on which place DNA methylation takes in the development of $A D$; whether it is involved in the onset of the $A D$ hallmarks or a downstream effect of these hallmarks. Nevertheless, the fact that different combination of mutations in "Alzheimer genes" result in different DNA methylation patters confirms that there is a link between Alzheimer and epigenetics.

\section{Role of the student}

Monique Havermans was a bachelor student working under the supervision of Daniel van den Hove and his PhD student Roy Lardenoije at the department of Neuropsychology when the research described in this report was performed. The topic was an ongoing project at the department. The lab work was done in Boston, USA by Roy Lardenoije, the analysis of the images was done by Monique Havermans under supervision of Roy Lardenoije and the processing of the data was performed by Roy Lardenoije but replicated by Monique Havermans. 


\section{References}

1. Bennett DA, Yu L, Yang J, Srivastava GP, Aubin C, De Jager PL. Epigenomics of Alzheimer's disease. TransI Res. 2015;165(1):200-20.

2. Xu X. DNA methylation and cognitive aging. Oncotarget. 2015.

3. Wang X, Michalis ML, Michaelis EK. Functional genomics of brain aging and Alzheimer's disease: focus on selective neuronal vulnerability. Curr Genomics. 2010;11(8):618-33.

4. Auclair G, Weber M. Mechanisms of DNA methylation and demethylation in mammals. Biochimie. 2012;94(11):2202-11.

5. Chouliaras L, van den Hove DL, Kenis G, et al. Age-related increase in levels of 5-hydroxymethylcytosine in mouse hippocampus is prevented by caloric restriction. Curr Alzheimer Res. 2012;9(5):536-44.

6. Lardenoije R, latrou A, Kenis G, et al. The epigenetics of aging and neurodegeneration. Progress in Neurobiology. 2015 [Epub ahead of print].

7. Coppieters N, Dieriks BV, Lill C, et al. Global changes in DNA methylation and hydroxymethylation in Alzheimer's disease human brain. Neurobiol Aging. 2014;35(6):1334-44. 